\title{
Renal MR angiography and perfusion in the pig using hyperpolarized water
}

\author{
Lipsø, Hans Kasper Wigh; Hansen, Esben Søvsø Szocska; Tougaard, Rasmus Stilling; Laustsen, \\ Christoffer; Ardenkjær-Larsen, Jan Henrik
}

Published in:

Magnetic Resonance in Medicine

Link to article, DOI:

$10.1002 / \mathrm{mrm} .26478$

Publication date:

2017

Document Version

Peer reviewed version

Link back to DTU Orbit

Citation (APA):

Lipsø, H. K. W., Hansen, E. S. S., Tougaard, R. S., Laustsen, C., \& Ardenkjær-Larsen, J. H. (2017). Renal MR angiography and perfusion in the pig using hyperpolarized water. Magnetic Resonance in Medicine, 78(3), 11311135. https://doi.org/10.1002/mrm.26478

\section{General rights}

Copyright and moral rights for the publications made accessible in the public portal are retained by the authors and/or other copyright owners and it is a condition of accessing publications that users recognise and abide by the legal requirements associated with these rights.

- Users may download and print one copy of any publication from the public portal for the purpose of private study or research.

- You may not further distribute the material or use it for any profit-making activity or commercial gain

- You may freely distribute the URL identifying the publication in the public portal

If you believe that this document breaches copyright please contact us providing details, and we will remove access to the work immediately and investigate your claim 
Title:

Renal MR Angiography and Perfusion in the Pig using Hyperpolarized Water

\section{Authors:}

Kasper Wigh Lipsø ${ }^{1}$, Esben Søvsø Szocska Hansen ${ }^{2,3}$, Rasmus Stilling Tougaard ${ }^{2,4}$, Christoffer Laustsen ${ }^{2}$ and Jan Henrik Ardenkjær-Larsen ${ }^{1,5}$

\section{Affiliations:}

${ }^{1}$ Department of Electrical Engineering, Technical University of Denmark, Kgs. Lyngby, Denmark.

${ }^{2}$ Department of Clinical Medicine, MR Research Centre, Aarhus University, Aarhus, Denmark

${ }^{3}$ Danish Diabetes Academy, Odense, Denmark

${ }^{4}$ Department of Cardiology - Research, Aarhus University Hospital, Aarhus, Denmark ${ }^{5}$ GE Healthcare, Brøndby, Denmark.

Corresponding author:

Jan Henrik Ardenkjær-Larsen, DTU Electrical Engineering, Ørsteds Plads, Building 349, room 126, 2800 Kgs. Lyngby, Denmark. Tel (0045) 4525 3918. E-mail: jhar@elektro.dtu.dk Word count: 2039

Running title: Renal MR Angiography and Perfusion in the Pig using Hyperpolarized Water Key words: Perfusion, Magnetic Resonance Angiography, Hyperpolarization, dissolution DNP 
Abstract

Purpose: To study hyperpolarized water as an angiography and perfusion tracer in a large animal model.

Methods: Protons dissolved in deuterium oxide $\left(D_{2} \mathrm{O}\right)$ were hyperpolarized in a SPINlab dissolution-DNP polarizer and subsequently investigated in vivo in a pig model at 3 T. Ca. $15 \mathrm{~mL}$ of hyperpolarized water was injected in the renal artery by hand over 4-5 s.

Results: A liquid state polarization of $5.3 \pm 0.9 \%$ of $3.8 \mathrm{M}$ protons in $15 \mathrm{~mL}$ of deuterium oxide was achieved with a $T_{1}$ of $24 \pm 1 \mathrm{~s}$. This allowed injection via an arterial catheter into the renal artery and subsequently high contrast imaging of the entire kidney parenchyma over several seconds. The dynamic images allow quantification of tissue perfusion, with a mean cortical perfusion of $504 \pm 123 \mathrm{~mL} / 100 \mathrm{~mL} / \mathrm{min}$.

Conclusion: Hyperpolarized water MR imaging was successfully demonstrated as a renal angiography and perfusion method. Quantitative perfusion maps of the kidney were obtained in agreement with literature and control experiments with gadolinium contrast.

Key words: Perfusion, Magnetic Resonance Angiography, Hyperpolarization, DNP 


\section{Manuscript Body}

\section{Introduction}

Magnetic Resonance Angiography (MRA) is an important tool for diagnosing various medical conditions such as emboli, stenoses and aneurysms. More specifically, renal artery MRA allows detection of renal vascular diseases such as fibromuscular dysplasia, venous thrombosis and arterial dissection (1). Sub-millimetre resolution can be obtained with proton imaging, and further optimization can be obtained by employing vascular contrast agents such as Gd-based blood pool agents (2) or nanoparticles (3). Despite significant improvement in scanner technology and reconstruction methods (4-6), MRA evaluation of small vessels is still challenging. This is mainly due to the inherent low sensitivity of MR, were the signal is proportional to the polarization of protons in the blood stream.

It has been demonstrated that this sensitivity problem can be overcome by hyperpolarizing nuclear spins (7). The method has recently been introduced in patients using $\left[1-{ }^{13} \mathrm{C}\right]$ pyruvate (8). By enhancing the polarization of ${ }^{13} \mathrm{C}$ by many orders of magnitude, the detected signal is increased dramatically, allowing in situ conversion of metabolic ${ }^{13} \mathrm{C}$ containing compounds to be imaged. The procedure of increasing polarization beyond thermal level, hyperpolarization (HP), can be achieved by dissolution Dynamic Nuclear Polarization (dDNP), where the high polarization of the high-gamma electrons, at cryogenic temperature and high magnetic field strength, is transferred to the nuclei by irradiation with microwaves. By rapid dissolution in a hot medium, the hyperpolarized nuclei can be brought to liquid state and injected into the patient before the polarization is lost due to longitudinal relaxation (7). Alternatively, metabolically inert ${ }^{13} \mathrm{C}$-labeled 
molecules can be used for angiographic imaging (9-11), as demonstrated in rodent and porcine models, and for perfusion of rodent organs $(12-15)$ or of tumor models $(16,17) .{ }^{13} \mathrm{C}$ or ${ }^{15} \mathrm{~N}$ labelled molecules for angiography and perfusion imaging exploits the long $T_{1}$ for selected ${ }^{13} \mathrm{C}$ and ${ }^{15} \mathrm{~N}$ molecules and the low signal background (18). Despite these advantages, these molecules exhibit partial permeability and active transport limitations.

A more accurate technique for water perfusion measurements has thus been called for $(19,20)$. A way to measure the perfusion of water molecules directly exists, using ${ }^{15} \mathrm{O}$ labelled water as a tracer for PET imaging $(21,22)$, with the main drawbacks being the use of a radio-active tracer and the limited spatial and temporal resolution of PET. Utilizing hyperpolarized water for angiographic and perfusion imaging overcomes several of these limitations. Water protons for imaging have previously been hyperpolarized by use of the Overhauser effect (23). However, the maximum achievable magnetization is limited. Typically, an enhancement of 100 times at $0.35 \mathrm{~T}$ can be obtained for pure water $\left(\mathrm{H}_{2} \mathrm{O}\right)$ corresponding to a polarization of $0.001 \%$. Higher polarizations have been reached with dissolution-DNP using the $3.35 \mathrm{~T}$ HyperSense polarizer, where $3-4 \mathrm{~mL}$ of $5 \mathrm{M}^{1} \mathrm{H}$ in $\mathrm{D}_{2} \mathrm{O}$ with a polarization of $3-5 \%$ have been applied to biomolecular NMR studies (24) or MR angiographies in the rat (25). Correcting for the difference in proton concentration, this corresponds to an available signal of two orders of magnitude larger than the Overhauser experiments. Imaging of hyperpolarized protons allows for the use of coils and pulse sequences already available in the clinical setting. Much more effort has gone into optimizing pulse sequences for optimal performance for proton imaging than for other nuclei. Moreover, the magnetization achievable with hyperpolarized water is superior to other nuclei, because of its large gyromagnetic ratio $\left(\gamma_{1 \mathrm{H}} \approx 4 \cdot \gamma_{13 \mathrm{C}}\right)$ and the potentially high proton concentration. As a tracer for perfusion, hyperpolarized water has additional 
interesting properties. Water diffuses freely in the vascular bed and intracellularly, and hence provides information not obtainable with larger compounds such as Gd-based MR contrast agents or larger nuclear tracers. Compared to the slow washout rate of conventional MR contrast agents (26), the $T_{1}$ value of hyperpolarized tracers hampers image acquisition, as well as it reduces the spatial range of measurable perfusion. However, the signal enhancement of hyperpolarization facilitates higher resolution and faster image acquisition, which allow us to trace the permeable protons in detail - with the stated limitations.

The purpose of this study was to demonstrate that hyperpolarized water can be used as a tracer to provide sub-second, high resolution angiographies in pigs by intra-arterial injection in the renal artery and that accurate perfusion maps can be obtained with good signal-to-noise ratio (SNR).

Methods

The polarizer and fluid path has been described in detail in (27). Briefly, the dDNP polarizer operates at $5 \mathrm{~T}$ and ca $0.9 \mathrm{~K}$. The sample and solvent was loaded into a fluid path that allows the sample to be polarized in the magnetic field at low temperature under microwave irradiation. The solvent was contained in a syringe that connects to the vial via two concentric tubes (inner and outer tube) and a valve. The syringe was placed in a heater/pressure module outside the polarizer cryostat. During dissolution the hyperpolarized solution leaves the polarizer through an exit tube.

Sample and solvent preparation: A sample of $1 \mathrm{~mL} 30 \mathrm{mM}$ TEMPO (2,2,6,6Tetramethylpiperidine 1-oxyl, 98\%, Sigma Aldrich, Denmark) in $\mathrm{H}_{2} \mathrm{O} /$ glycerol 1:1 (w/w) was prepared. The sample was placed in the fluid path vial and frozen in liquid nitrogen. 
Dissolution medium (DM) consisted of $\mathrm{D}_{2} \mathrm{O}$ with $1 \mathrm{mM}$ calcium disodium ethylenediaminetetraacetic acid (EDTA) and $9 \mathrm{~g} / \mathrm{L} \mathrm{NaCl}$ (both Sigma Aldrich, Denmark).

$31.01 \mathrm{~g} \mathrm{DM}(28 \mathrm{~mL})$ was filled in the syringe and degassed by bubbling with helium gas for 10 minutes. The fluid path was flushed with helium gas for 2 minutes in order to remove air and ensure clear passage.

Polarization: The sample vial was rapidly transferred from the liquid nitrogen bath to the 5 T magnet in the polarizer (SPINlab, GE Healthcare, Brøndby, Denmark). The sample was irradiated with app. $50 \mathrm{~mW}$ microwaves at $139.923 \mathrm{GHz}$ for one hour.

Dissolution: The syringe containing the DM was heated to $130{ }^{\circ} \mathrm{C}$ for one hour before dissolution while being under pressure. The piston of the syringe was driven with 16 bar pressure during dissolution. The dissolved sample was injected via the exit tube into a separatory funnel (100 mL) containing $25 \mathrm{~mL}$ of heptane (>99\%, Sigma Aldrich, Denmark) to extract the radical from the aqueous phase. The heptane had been degassed by flushing with helium gas in the funnel for 10 minutes. The funnel was shaken heavily during dissolution for a total of $10 \mathrm{~s}$ from dissolution start. The lower, aqueous phase was extracted into a syringe before transferring to the scanner room. The proton concentration in the dissolved sample was analyzed by NMR after addition of $2 \mathrm{M}$ formate solution as reference.

Animal protocol: All animal experiments were carried out in compliance with the guidelines for use and care of laboratory animals and were approved by the Danish Inspectorate of Animal Experiments. Four healthy $30 \mathrm{~kg}$ female Danish domestic pigs were pre-sedated with intramuscular injection of Stressnil $(1 \mathrm{~mL} / \mathrm{kg})$, and Midazolam $(1 \mathrm{~mL} / \mathrm{kg})$, and anesthesia was maintained by continuous infusion of Propofol $(0.4 \mathrm{mg} / \mathrm{kg} / \mathrm{h})$ and Fentanyl 
(8 $\mathrm{\mu g} / \mathrm{kg} / \mathrm{h})$. The animals were intubated and mechanically ventilated with a $60 \% \mathrm{O}_{2}$-air mix.

A $6 \mathrm{~F}$ introducer sheath was positioned in the left common femoral artery by ultrasound guidance to allow access for a $6 \mathrm{~F}$, JR 4.0, angiography catheter to be placed in the left main renal artery via x-ray guidance. The angiography catheter was repeatedly flushed with heparinized sterile water to avoid occlusion. $15 \mathrm{~mL}$ HP water was injected over $5 \mathrm{~s}$, initiated approximately $22 \mathrm{~s}$ after dissolution.

Through a vein access in the right ear, a bolus of $0.2 \mathrm{mmol} / \mathrm{kg}$ Gd-DTPA (Gadobutrol, Gadovist, Bayer Schering Pharma, Berlin) was administered manually for conventional $\mathrm{T}_{1^{-}}$ DCE-MRI.

MRI acquisition: MRI detection was performed on a 3 T GE HDx with an 8-channel cardiac array receiver coil (GE Healthcare, Milwaukee, WI, USA). Phantom experiments were performed with a gradient echo sequence using a flip angle of $1^{\circ}$, matrix size $128 \times 128$, $\mathrm{FOV}=(24 \mathrm{~mm})^{2}$ in a slab of $40 \mathrm{~mm}$ with $\mathrm{TR}=3 \mathrm{~ms}, \mathrm{TE}=0.784 \mathrm{~ms}$. The acquisition time of each frame was $450 \mathrm{~ms}$, and images were acquired with a time delay of one second.

Axial and coronal images were acquired to cover both kidneys to allow for planning of the angiography slice for hyperpolarized water imaging. The planning sequence was as follows: a $3 \mathrm{D} \mathrm{T}_{1}$ weighted sequence with a standard steady state free precession sequence (echo time (TE) $1.1 \mathrm{~ms}$, repetition time (TR) $2.7 \mathrm{~ms}$, flip angle (FA) $35^{\circ}$, image matrix 256x256, field of view (FOV) $340 \times 340 \mathrm{~mm}^{2}$, in-plane resolution $1.3 \mathrm{~mm}$, slice thickness $3 \mathrm{~mm})$.

Angiographies were acquired in the coronal plane of the left kidney using a gradient echo sequence with $5^{\circ} \mathrm{FA}$, slice thickness $40 \mathrm{~mm}$, TR $=3.4 \mathrm{~ms}$, TE $=0.984 \mathrm{~ms}, 256 \times 256$ 
matrix, FOV $=140 \times 140 \mathrm{~mm}^{2}$. The acquisition time was $870 \mathrm{~ms}$. The Gd-perfusion sequence was executed with the following parameters: spoiled gradient echo, inversion time $=850 \mathrm{~ms}, 10^{\circ} \mathrm{FA}$, slice thickness $50 \mathrm{~mm}, \mathrm{TR}=2 \mathrm{~s}, \mathrm{TE}=1.4 \mathrm{~ms}, 128 \times 128$ matrix, $\mathrm{FOV}=290 \times 290 \mathrm{~mm}^{2}$.

MRI post processing: Phantom data were analyzed using MATLAB (Mathworks, Natick, MA, USA). Renal perfusion maps were calculated in OsiriX (Pixmeo, Geneva, Switzerland) using an open source OsiriX plug-in for DCE-MRI perfusion analysis (29). A pixel-by-pixel model-free fast deconvolution was applied, assuming a direct signal enhancement of the hyperpolarized water, a hematocrit of 0.45 and a regularization of 0.15 . The hyperpolarized arterial input function (AIF) was fixed at the end of the arterial bolus ( $7 \mathrm{~s}$ in Figure 2), to avoid the injection characteristics of the AIF. The Gd-T 1 -DCE contrast was similarly analysed by a pixel-by-pixel model-free fast deconvolution, assuming a relative signal enhancement accounting for the signal modulations by the contrast agent.

Results

The average proton concentration in the dissolved sample was $3.84 \pm 0.22 M(n=10) .18 \pm 1$ $\mathrm{mL}$ of dissolved sample was produced. The polarization was measured by placing a syringe with dissolved hyperpolarized sample next to a similar syringe of saline and image a slab of $40 \mathrm{~mm}$. The signal obtained from the phantoms is plotted in Figure 1. The initial hyperpolarized signal was 211 times the mean signal in the thermal phantom of Boltzmann polarization $10.5 \mathrm{ppm}$. Thus, after correction for the proton concentration, the polarization was $5.3 \pm 0.9 \%(n=4) . A T_{1}$ of $24 \pm 1 s$ was measured.

Angiographies of the arterial system of the left kidney were produced with hyperpolarized water in 4 animals (Figure 2). Perfusion maps were calculated for the four animals (Figure 
3). A mean cortical perfusion of $504 \pm 123 \mathrm{~mL} / 100 \mathrm{~mL} / \mathrm{min}$ was found. Some folding of the signal from the catheter appears in the images in Figure 3C and 3D. The arterial input function is shown in Figure 2 along with the cortical signal curve. The arterial input function is heavily modulated by the direct injection, as seen by the flat top of the input from 3-7 s. Trimming the AIF to the end of the injection, was found to reproducibly reflect the average perfusion found by Gd-T1-DCE analysis of $450 \mathrm{~mL} / 100 \mathrm{~mL} / \mathrm{min}(\mathrm{N}=2)$.

Discussion

The main finding of this study was the demonstration of a feasible method for acquiring reproducible renal angiograms and perfusion maps using hyperpolarized water with both high resolution and high signal to noise ratio in a large animal model resembling human renal anatomy and physiology. The produced volume of $15 \mathrm{~mL}$ exceeds the previously demonstrated volumes of $\sim 4 \mathrm{~mL}$ produced on the HyperSense (25), and the SPINlab and fluid path technology facilitates sterile samples for clinical applications (10). Good agreement was found between the calculated hyperpolarized water perfusion and both the reported Gd-based perfusion values in healthy porcine models (30) and with our Gd-based perfusion measurements (average of $450 \mathrm{~mL} / 100 \mathrm{~mL} / \mathrm{min}$ ). Further optimization of MRI sequence, sample preparation, radical extraction as well as automation and consistency of the injection process are likely to enhance image quality and applicability further. A longer bolus injection to approximate a Heaviside input function may improve accuracy. The primary limitation is the short lifetime of the hyperpolarized water, reducing its applicability for highly perfused organs. The use of direct arterial injection imposes challenges on both animal handling (future clinical usability) and on the modeling of the perfusion information. The angiograms are of excellent quality; however, Gd-based and non-contrast angiography of the renal arteries is currently providing sufficient diagnostic quality. On the 
other hand, since the discovery of Nephrogenic Systemic Fibrosis, new MR methods to measure perfusion may have a role. In addition to the specific extravasation properties of water relative to any other molecule, the signal that can be created by hyperpolarized ${ }^{1} \mathrm{H}$ exceeds any other magnetic nucleus (e.g. $\left.{ }^{13} \mathrm{C}\right)$.

Both the radical and molecular oxygen in the sample induce dipolar relaxation that reduces the longitudinal relaxation time $T_{1}$. Thus, the radical extraction in the organic phase as well as the oxygen removal during preparation are crucial elements for achieving long $T_{1}$ and, ultimately, high polarization after substance transferring. TEMPO was chosen without optimization as a good compromise between water solubility and preferential partitioning to the heptane phase.The proton concentration was chosen as an optimal compromise between limitation of the intermolecular dipolar relaxation between proton nuclei and achievement of high signal in the injectable sample.

The ability to hyperpolarize water protons in a polarizer designed for clinical use, with sufficient polarization to allow ultra-fast angiographic and perfusion assessment, shows great promise for future clinical translation.

\section{Acknowledgements}

Danish Research Council grant 12-127232. Danish National Research Foundation, DNRF124. 


\section{References}

1. Attenberger UI, Morelli JN, Schoenberg SO, Michaely HJ. Assessment of the kidneys: magnetic resonance angiography, perfusion and diffusion. J Cardiovasc Magn Reson 2011;13:1-13.

2. Kinner S, Maderwald S, Parohl N, Albert J, Corot C, Robert P, Barkhausen J, Vogt FM. Contrast-enhanced magnetic resonance angiography in rabbits: evaluation of the gadolinium-based agent p846 and the iron-based blood pool agent p904 in comparison with gadoterate meglumine. Invest Radiol 2011;46:524-529.

3. Howles GP, Ghaghada KB, Qi Y, Mukundan S, Johnson GA. High-resolution magnetic resonance angiography in the mouse using a nanoparticle blood-pool contrast agent. Magn Reson Med 2009;62:1447-1456.

4. Addy NO, Ingle RR, Wu HH, Hu BS, Nishimura DG. High-resolution variable-density 3D cones coronary MRA. Magn Reson Med 2015;74:614-621.

5. Koktzoglou I, Murphy IG, Giri S, Edelman RR. Quiescent interval low angle shot magnetic resonance angiography of the extracranial carotid arteries. Magn Reson Med 2016;75:2072-2077.

6. Nezafat M, Henningsson M, Ripley DP, Dedieu N, Greil G, Greenwood JP, Börnert P, Plein S, Botnar RM. Coronary MR angiography at 3T: fat suppression versus water-fat separation. Magn Reson Mater Physics Biol Med 2016; doi: 10.1007/s10334-016-0550-7. [Epub ahead of print]

7. Ardenkjær-Larsen JH, Fridlund B, Gram A, Hansson G, Hansson L, Lerche MH, Servin R, Thaning M, Golman K. Increase in signal-to-noise ratio of > 10,000 times in liquid-state NMR. Proc Natl Acad Sci U S A 2003;100:10158-10163. 
8. Nelson SJ, Kurhanewicz J, Vigneron DB, et al. Metabolic Imaging of Patients with Prostate Cancer Using Hyperpolarized [1-13 C] Pyruvate. Sci Transl Med 2013:5;198ra108.

9. Golman K, Axelsson O, Jóhannesson H, Månsson S, Olofsson C, Petersson JS, Parahydrogen-induced polarization in imaging: Subsecond 13C angiography. Magn Reson Med 2001;46:1-5.

10. Svensson J, Månsson S, Johansson E, Petersson JS, Olsson LE. Hyperpolarized 13C MR angiography using trueFISP. Magn Reson Med 2003;50:256-262.

11. Lipsø KW, Magnusson P, Ardenkjær-Larsen JH. Hyperpolarized 13 C MR Angiography. Curr Pharm Des 2016;22:90-95.

12. Qi H, Nørlinger TS, Nielsen PM, Bertelsen LB, Mikkelsen E, Xu Y, StødkildeJørgensen $\mathrm{H}$, Laustsen $\mathrm{C}$. Early diabetic kidney maintains the corticomedullary urea and sodium gradient. Physiol Rep 2016;4:1-6.

13. Lau AZ, Miller JJ, Robson MD, Tyler DJ. Cardiac perfusion imaging using hyperpolarized $13 \mathrm{c}$ urea using flow sensitizing gradients. Magn Reson Med 2016;75:1474-1483.

14. Lau AZ, Miller JJ, Robson MD, Tyler DJ. Simultaneous assessment of cardiac metabolism and perfusion using copolarized [1-(13) C]pyruvate and (13) C-urea. Magn Reson Med 2016; doi: 10.1002/mrm.26106. [Epub ahead of print].

15. Von Morze C, Bok RA, Reed GD, Ardenkjær-Larsen JH, Kurhanewicz J, Vigneron DB. Simultaneous multiagent hyperpolarized 13C perfusion imaging. Magn Reson 
Med 2014;72:1599-1609.

16. Park I, von Morze C, Lupo JM, Ardenkjær-Larsen JH, Kadambi A, Vigneron DB, Nelson SJ. Investigating tumor perfusion by hyperpolarized ${ }^{13} \mathrm{C}$ MRI with comparison to conventional gadolinium contrast-enhanced MRI and pathology in orthotopic human GBM xenografts. Magn Reson Med 2016. doi: 10.1002/mrm.26155. [Epub ahead of print].

17. Von Morze C, Larson PEZ, Hu S, Yoshihara HAI, Bok RA, Goga A, ArdenkjærLarsen JH, Vigneron DB. Investigating tumor perfusion and metabolism using multiple hyperpolarized 13C compounds: HP001, pyruvate and urea. Magn Reson Imaging 2012;30:305-311.

18. Durst M, Chiavazza E, Haase A, Aime S, Schwaiger M, Schulte RF, $\alpha$ trideuteromethyl[15N]glutamine: A long-lived hyperpolarized perfusion marker. Magn Reson Med 2016. doi: 10.1002/mrm.26104. [Epub ahead of print].

19. Bergmann SR, Herrero P, Markham J, Weinheimer CJ, Norine M. Noninvasive Quantitation of Myocardial Blood Flow in Human Subjects With Oxygen-15 Labeled Water and Positron Emission Tomography. J Am Coll Cardiol 1989;14:639-652.

20. De Langen AJ, van den Boogaart VEM, Marcus JT, Lubberink M. Use of H2(15)OPET and DCE-MRI to measure tumor blood flow. Oncologist 2008;13:631-644.

21. Herscovitch P, Markham J, Raichle ME. Brain Blood Flow Measured with Intravenous H215O.: I. Theory and Error Analysis. J Nucl Med 1983;24:782-789.

22. Raichle ME, Martin WRW, Herscovltch P, Mintun MA, Markham J. Brain Blood Flow Measured with Intravenous H215O.: II. Implementation and Validation. J Nucl Med 1983;24:790-798. 
23. McCarney ER, Armstrong BD, Lingwood MD, Han S. Hyperpolarized water as an authentic magnetic resonance imaging contrast agent. Proc Natl Acad Sci U S A 2007;104:1754-1759.

24. Harris T, Szekely O, Frydman L. On the potential of Hyperpolarized Water in Biomolecular NMR Studies. J Phys Chem B 2014;118:3281-3290.

25. Ardenkjær-Larsen JH, Laustsen C, Bowen S, Rizi R. Hyperpolarized H2O MR angiography. Magn Reson Med 2014;71:50-56.

26. Schoenberg SO, Rieger J, Weber $\mathrm{CH}$, Michaely HJ, Waggershauser $\mathrm{T}$, Ittrich $\mathrm{C}$, Dietrich O, Reiser MF. High-Spatial-Resolution MR Angiography of Renal Arteries with Integrated Parallel Acquisitions: Comparison with Digital Subtraction Angiography and US. Radiology 2005;235:687-698.

27. Ardenkjær-Larsen JH, Leach AM, Clarke N, Urbahn J, Anderson D, Skloss TW. Dynamic nuclear polarization polarizer for sterile use intent. NMR Biomed $2011 ; 24: 927-932$.

28. Zöllner FG, Weisser G, Reich M, Kaiser S, Schoenberg SO, Sourbron SP, Schad LR. Ummperfusion: An open source software tool towards quantitative mri perfusion analysis in clinical routine. J Digit Imaging 2013;26:344-352.

29. Wentland AL, Artz NS, Fain SB, Grist TM, Djamali A, Sadowski EA. MR measures of renal perfusion, oxygen bioavailability and total renal blood flow in a porcine model: Noninvasive regional assessment of renal function. Nephrol Dial Transplant 2012;27:128-1352. 


\section{Figure Legends}

Figure 1: The graph shows ROI signal means of the hyperpolarized ${ }^{1} \mathrm{H}$ in $\mathrm{D}_{2} \mathrm{O}$ (green, decaying signal) and the $\mathrm{H}_{2} \mathrm{O}$ thermal phantoms (blue, steady signal). The phantoms consist of two syringes inside a loading phantom (ring structure). Images (individually scaled) are shown at 0 and 200 s. The hyperpolarized MR signal is initially 211 times stronger than the thermal $\mathrm{H}_{2} \mathrm{O}$ signal, and the $T_{1}$ of the decay is $24 \mathrm{~s}$. The hyperpolarized

signal decays into the noise since the ${ }^{1} \mathrm{H}$ density is ca 20 times lower than the thermal $\mathrm{H}_{2} \mathrm{O}$ sample.

Figure 2: Image intensity in the arterial input ROI and cortex ROI for the same pig. Images visualize time steps at 3, 5, 7 and 9 s.

Figure 3: $A, B, C, D$ show perfusion maps generated from four individual pigs with a mean cortical perfusion of $504 \pm 123 \mathrm{~mL} / 100 \mathrm{~mL} / \mathrm{min} . \mathrm{C}$ is the same pig as shown in Figure 2. 
Figures

Figure 1

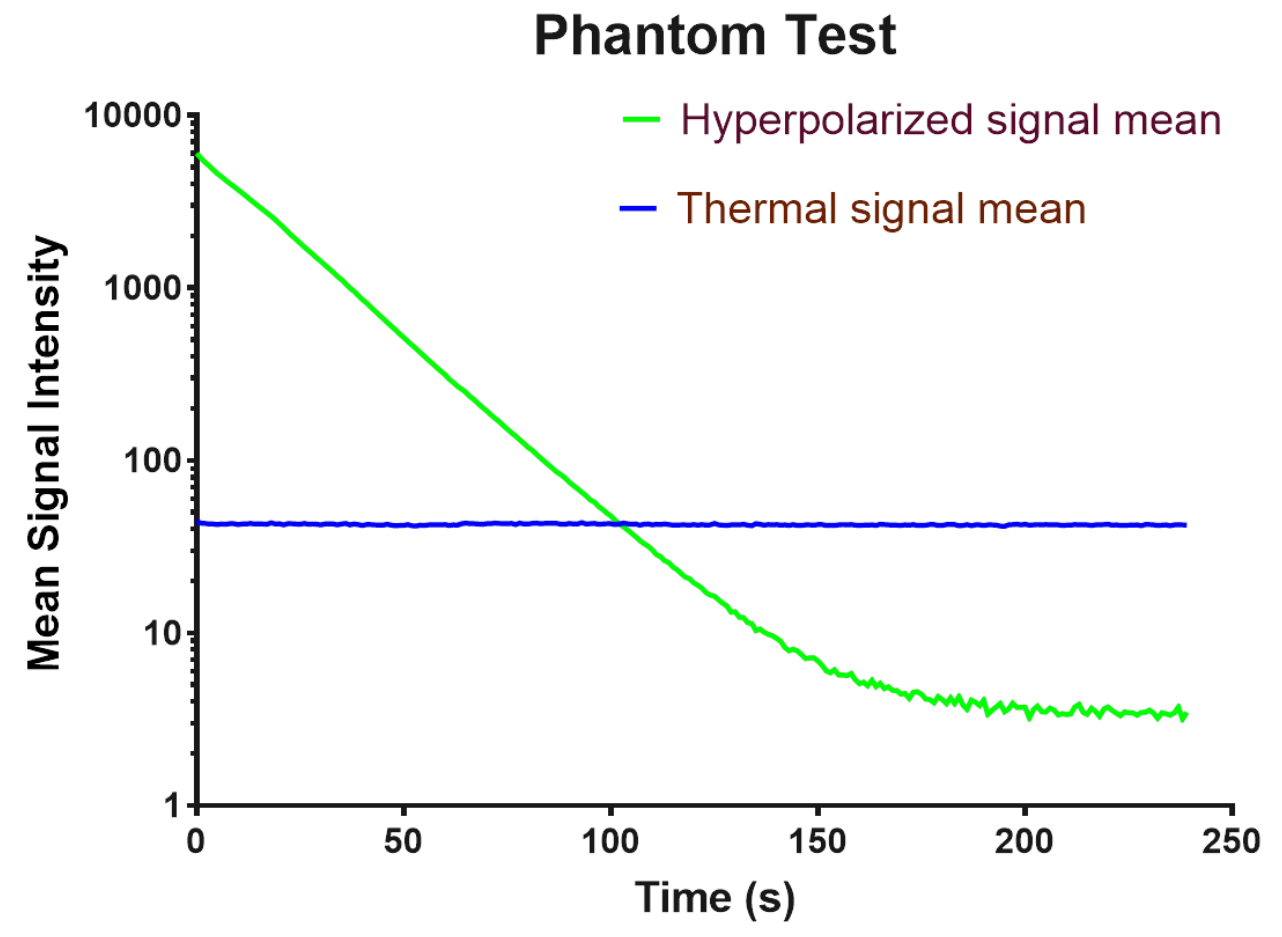

$0 \mathrm{~s}$

00

$200 s$

- 0 
Figure 2

Time Series
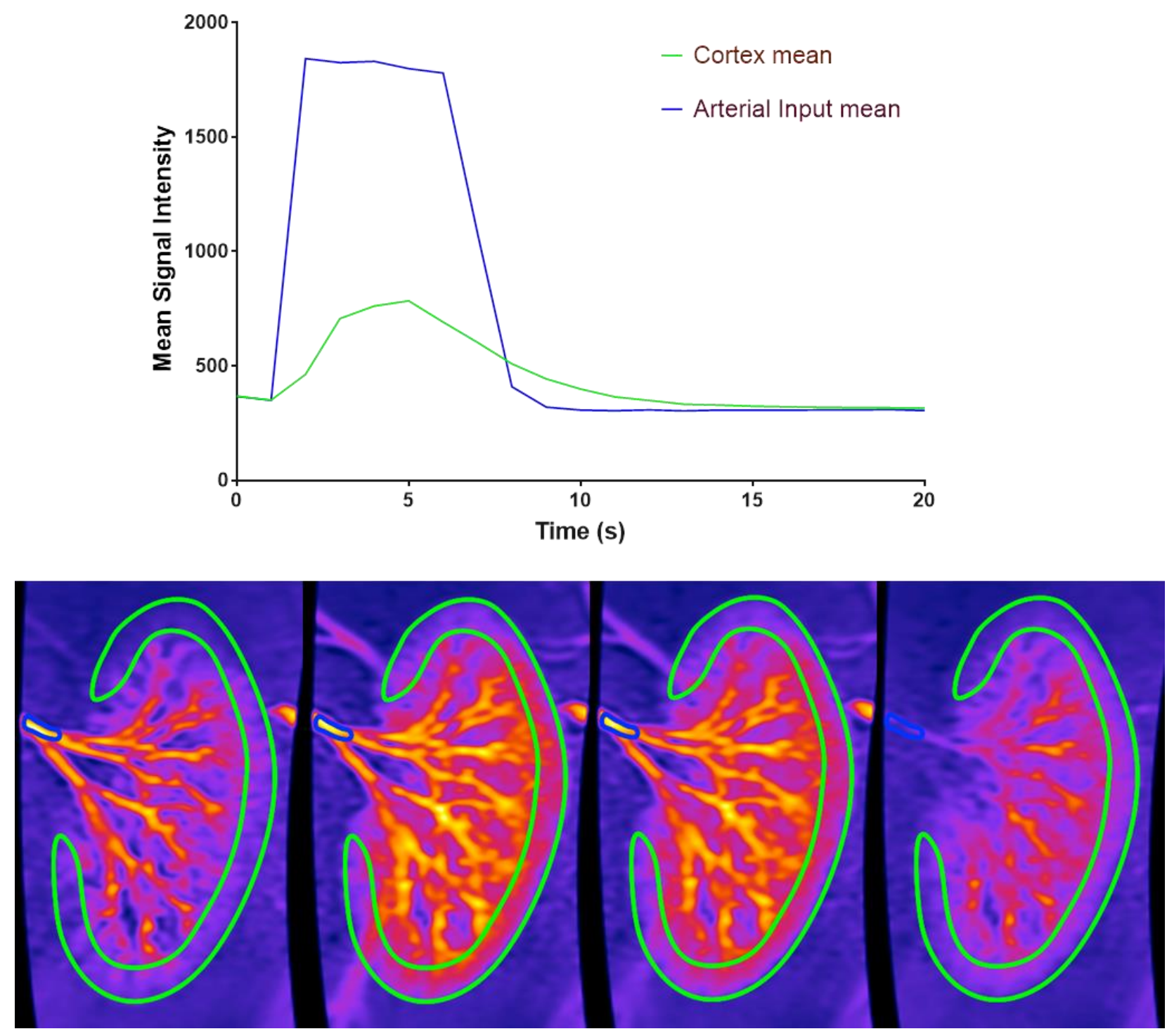
Figure 3

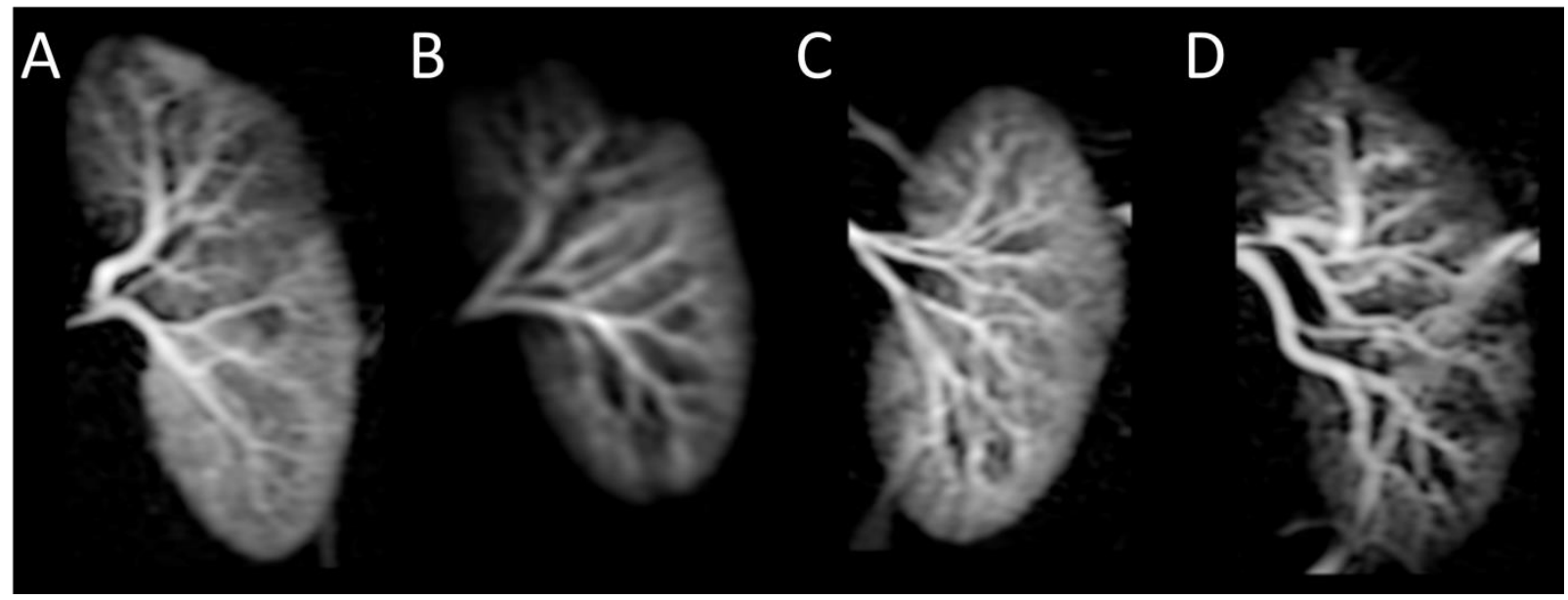

